Citation: P. Deguy, M. Ripepe, G. Lacanna, L. Orti (2021). Geodatabase per la valutazione speditiva a larga scala della vulnerabilità sismica di un'area urbana complessa: applicazione alla città di Firenze. Bollettino della Società Geografica Italiana serie 14, 4(2): 79-87. doi: 10.36253/bsgi-1453

Copyright: @ 2021 P. Deguy, M. Ripepe, G. Lacanna, L. Orti. This is an open access, peer-reviewed article published by Firenze University Press (http:// www.fupress.com/bsgi) and distributed under the terms of the Creative Commons Attribution License, which permits unrestricted use, distribution, and reproduction in any medium, provided the original author and source are credited.

Data Availability Statement: All relevant data are within the paper and its Supporting Information files.

Competing Interests: The Author(s) declare(s) no conflict of interest.
Note di ricerca

\section{Geodatabase per la valutazione speditiva a larga scala della vulnerabilità sismica di un'area urbana complessa: applicazione alla città di Firenze}

\section{Geodatabase for the large-scale seismic vulnerability assessment of a complex urban area: application to the city of Florence}

\author{
Pauline Deguy ${ }^{1}$, Maurizio Ripepe ${ }^{2}$, Giorgio Lacanna ${ }^{2}$, Letizia Orti ${ }^{2}$ \\ ${ }^{1}$ Dipartimento di Storia, Archeologia, Geografia, Arte e Spettacolo, Università degli Studi \\ di Firenze, Firenze, Italia \\ ${ }^{2}$ Dipartimento di Scienze della Terra, Università degli Studi di Firenze, Firenze, Italia \\ E-mail: pauline.deguy@unifi.it, maurizio.ripepe@unifi.it, giorgio.lacanna@unifi.it, leti- \\ zia.orti@unifi.it
}

\begin{abstract}
The seismic vulnerability assessment of a city is usually based on buildings' characteristics, underground geophysical properties, socio-economic activities, and population density. This research highlights the value of a complete and manageable database for large-scale seismic vulnerability assessment of urban areas: such databases are often missing or partially developed for the majority of Italian cities. This study is based on the creation of a GIS database containing useful information to identify and valuate some indicators of seismic vulnerability in order to understand an earthquake's effect for the actual configuration of a city. This system could be a growing source of information on cities and, if used in the proper way, a useful tool for monitoring, for seismic vulnerability assessment, and for spatial urban planning. The experience gained in this field of application can be transferred to other situations, both for the procedures and the workflow system.
\end{abstract}

Keywords: seismic vulnerability, GIS, Florence.

Riassunto. La vulnerabilità sismica di una città si valuta generalmente in base alle caratteristiche degli edifici, alle proprietà geofisiche del sottosuolo, alle attività socioeconomiche e alla densità abitativa. Il presente studio solleva il problema dell'importanza di un database completo e fruibile per la valutazione a larga scala della vulnerabilità sismica di uno "spazio urbano"1, molto spesso mancante o quanto meno incompleto per una gran parte delle città italiane. Questo lavoro si è basato sulla creazione di un database GIS completo di informazioni utili alla definizione e alla rappresentazione di una serie di indicatori di vulnerabilità sismica al fine di comprendere le conseguenze di un terremoto sull'organizzazione attuale di una città. Tale sistema rappresenta una

\footnotetext{
${ }^{1}$ Definito da Bertin $(2015,62)$ come il risultato dell'interazione tra 3 livelli: la comunità umana eterogenea, ledilizia pubblica e privata e l'ordinativo
} 
fonte crescente di informazioni sulla città che, se analizzate in maniera appropriata, possono costituire sia uno strumento di monitoraggio che di conoscenza della vulnerabilità sismica e di conseguenza anche uno strumento di pianificazione. L'esperienza maturata in questo contesto applicativo può essere trasferita ad altre situazioni, sia per le tecnologie utilizzate che per i workflow sperimentati.

Parole chiave: vulnerabilità sismica, GIS, Firenze.

\section{Introduzione}

Il presente studio propone una metodologia per la valutazione della vulnerabilità sismica urbana della città di Firenze attraverso l'uso della tecnologia GIS allo scopo di creare un sistema informativo del patrimonio edilizio (residenziale, pubblico e produttivo) e sulla popolazione (residente o turistica). I dati sono stati ottenuti da studi precedenti sulla geologia e la sismologia dell'area (Coli et al. 2008), da ISTAT e altri strati informativi messi a disposizione dal Comune di Firenze oltre che da rilievi sismici in-situ su un campione rappresentativo dell'edificato fiorentino al fine di definirne il periodo naturale di oscillazione (Ripepe et al. 2015).

Il rischio è un parametro funzione della probabilità di accadimento che può essere descritto dalla seguente formula:

\section{RISCHIO = PERICOLOSITÀ * VULNERABILITÀ * VALORE ESPOSTO ${ }^{2}$}

Con pericolosità (o hazard) si intende "la probabilità che un tipo di evento di una determinata intensità si verifichi in un luogo specifico in un intervallo di tempo prefissato" (Varnes et al. 1984), detto questo, possiamo affermare che la pericolosità dipende fortemente sia dalle caratteristiche della sorgente di rischio sia da quelle del sito al quale è riferita. Applicata al rischio sismico, la pericolosità è una caratteristica del territorio indipendente dalla presenza o meno di un bene su di esso, è valutata basandosi sulla storia e sulle caratteristiche sismologiche, geografiche e geologiche.

La vulnerabilità o la propensione di persone, beni o attività a subire danni in seguito ad un determinato evento avverso, si misura in scala o in soglie. Da un

\footnotetext{
${ }^{2}$ Formula approvata dalla Comunità Europea nel 1996 che la definisce come "una funzione dell'ampiezza del possibile danno che può risultare dal pericolo considerato e della probabilità di accadimento di quel danno (a sua volta funzione della frequenza e durata dellesposizione, della probabilità di accadimento dell'evento pericoloso e della possibilità di evitare o limitare il danno)".
}

punto di vista socio-culturale, la geografa Susan Cutter definisce la vulnerabilità come "la probabilità che un soggetto o un gruppo umano possa essere esposto e colpito negativamente da un pericolo. È l'interazione tra il pericolo dei luoghi e il profilo sociale della comunità" (Cutter et al. 2003).

Il valore esposto definisce la misura di tutto ciò che può essere perso a seguito del verificarsi di un evento avverso in una determinata area. Questo valore può essere espresso in vite umane oppure in termini economici o in beni. Esso è quindi dipendente sia dalle attività sia dalle peculiarità ambientali che caratterizzano l'area di riferimento.

La vulnerabilità sismica di una struttura si misura dunque sia in termini di perdita o riduzione di efficienza, sia come la capacità residua di e assicurare le funzioni che normalmente svolge a regime. L'esposizione di un'area interessata dal rischio sismico, è data da molteplici elementi: per conoscere il valore esposto di un territorio è necessario conoscere la quantità e la qualità degli elementi antropici presenti e potenzialmente a rischio. In generale, per semplificare la definizione dell'esposizione di un'area, si ripartiscono gli elementi esposti in categorie alle quali vengono attribuite un danno fisico occorso, un'entità e un valore delle perdite preventivate per un determinato evento.

\section{Il database}

La creazione di un database utile alla valutazione del rischio sismico necessità l'integrazione di informazioni tecniche (proprietà geofisiche del sottosuolo, caratteristiche strutturali degli edifici) e di dati socio-economici (destinazione o classe di uso degli edifici, numero di abitanti e la loro età, affluenza turistica per gli edifici di maggior attrattività).

Dai dati urbanistici del Comune di Firenze (aggiornati al 2016) e dai dati ISTAT risultano censiti nel territorio comunale più di 47.500 edifici; ad ognuno di questi sono state associate informazioni raccolte durante rilievi sismici e architettonico-strutturali in-situ allo scopo di creare una banca dati relativa alle caratteristiche tecniche degli edifici fiorentini. Per ogni edificio il database contiene le seguenti informazioni: altezza, materiale e anno di costruzione, periodo di oscillazione naturale dell'edificio (Ripepe et al. 2015), caratteristiche fisiche del sottosuolo (fattore di amplificazione o effetto di sito, periodo di oscillazione delle onde sismiche), classe di uso $^{3}$, indice di risonanza (Ripepe et al. 2015) che

\footnotetext{
3 "Con riferimento alle conseguenze di una interruzione di operatività o
} di eventuale collasso, le costruzioni sono suddivise in classe d'uso", cap. 
Tabella 1. Tabella riassuntiva dei dati raccolti e dei metodi di analisi o di estrazione dei dati.

\begin{tabular}{|c|c|c|}
\hline Informazione & Fonti & Metodologia di analisi o di estrazione dei dati \\
\hline Altezza & - Ufficio Urbanistica, Comune di Firenze & Calcolo della differenza tra DSM e DTM \\
\hline Anno di costruzione & $\begin{array}{l}\text { - Ufficio Urbanistica, Comune di Firenze } \\
\text { - Censimento ISTAT }\end{array}$ & Estrazione dei dati presenti nelle 2 fonti \\
\hline Materiale & $\begin{array}{l}\text { - Ufficio Urbanistica, Comune di Firenze } \\
\text { - Censimento ISTAT }\end{array}$ & $\begin{array}{l}\text { Attribuzione in funzione dellanno di } \\
\text { costruzione e estrazione dei dati presenti nel } \\
\text { database ISTAT }\end{array}$ \\
\hline Periodo di oscillazione delledificio & $\begin{array}{l}\text { - Rilievi in-situ } \\
\text { - Normativa per le costruzioni }\end{array}$ & $\begin{array}{l}\text { Attribuzione di un valore di periodo in } \\
\text { funzione del materiale di costruzione }\end{array}$ \\
\hline $\begin{array}{l}\text { Caratteristiche del sottosuolo in particolare il } \\
\text { periodo }\end{array}$ & $\begin{array}{l}\text { - Dipartimento di Scienze della Terra, } \\
\text { Università di Firenze }\end{array}$ & Estrazione dei dati raster ai singoli edifici \\
\hline Classe di uso & $\begin{array}{l}\text { - Censimento ISTAT } \\
\text { - Normativa per le costruzioni }\end{array}$ & Estrazione dei dati ad ogni singolo edificio \\
\hline Indice di risonanza & $\begin{array}{l}\text { - Dipartimento Scienze della Terra, } \\
\text { Università di Firenze } \\
\text { - Rilievi in-situ } \\
\text { - Normativa per le costruzioni }\end{array}$ & $\begin{array}{l}\text { Calcolo, per ogni singolo edificio, del rapporto } \\
\text { tra periodo dell'edificio e quello del sottosuolo }\end{array}$ \\
\hline Dati demografici & - Censimento ISTAT & Estrazione dei dati per isolato \\
\hline
\end{tabular}

si ottiene grazie al rapporto tra periodo di oscillazione dell'edificio e quello del sottosuolo sottostante (Tab. 1). L'uso principale degli edifici può essere diviso in 6 classi: residenziale, turismo/beni culturali, commerciale, direzionale, produttivo, amministrazione/servizi. Conviene specificare che gli edifici sono stati classificati secondo il loro uso principale, ad esempio alcuni beni culturali sono ormai utilizzati come scuole, biblioteche, ecc. Tutte queste informazioni sono state integrate per 37.523 edifici, ovvero per il $79 \%$ dell'edificato totale.

\subsection{La popolazione residenziale e turistica}

Integrando i dati dell'Ufficio di Statistica dell'area fiorentina e del Comune di Firenze, sono state aggiunte alla banca dati così ampliata degli edifici, informazioni sul numero di residenti censiti per isolato. La destinazione d'uso degli edifici è un dato rilevante per la definizione del livello di esposizione umano in quanto permette di differenziare quelli con una presenza umana più elevata durante il giorno, nel caso di edifici ad uso amministrativo, commerciale e beni culturali, da quelli ad uso residenziale, nei quali la presenza diventa più significativa durante le ore notturne (Fig. 1).

Firenze è una città caratterizzata da una forte attrazione turistica, pertanto il flusso turistico deve far parte integrante del valore esposto nella formula del rischio. A questo scopo, sono state aggiunte, all'interno della ban-

2.4.2 delle Norme Tecniche per le costruzioni 2018 (NTC 2018) ca dati, informazioni riguardanti il flusso di visitatori all'interno dei musei e dei beni culturali fiorentini (ottenuto dai dati Istat 2011).

\subsection{Il patrimonio edilizio}

Come già detto, il numero degli edifici del territorio comunale fiorentino risultante dal Censimento ISTAT 2011 è di 47.509. Per quanto riguarda la destinazione d'uso, il "Residenziale" rappresenta circa il 65\% ed è maggiormente presente nelle aree periferiche, mentre nel centro storico prevalgono gli edifici a destinazione "Commerciale" e "Terziario". La maggioranza dei fabbricati a destinazione "Produttiva" si trova nella zona settentrionale del territorio comunale (Quartiere 5 Rifredi), così come gli edifici di più recente costruzione; nella zona orientale (Quartiere 4 Isolotto-Legnaia) invece, si trovano prevalentemente edifici costruiti prima del 1945. Nel centro storico, si concentrano strutture realizzate prima del 1833. La tecnica costruttiva prevalente nella città è la "Muratura portante", mentre il "Calcestruzzo armato" (CA), tipico degli edifici più recenti, è maggiormente presente nella zona orientale (Quartiere 4 Isolotto-Legnaia). A partire da questi dati, gli edifici sono stati classificati ed è stata effettuata una prima omogeneizzazione delle tipologie edilizie (Fig. 2):

a) pre-1833: muratura piena a pietra e/o mattoni con fondazioni dirette (23\%);

b) dal 1833 al 1923: muratura a sacco con fondazione diretta (22\%); 


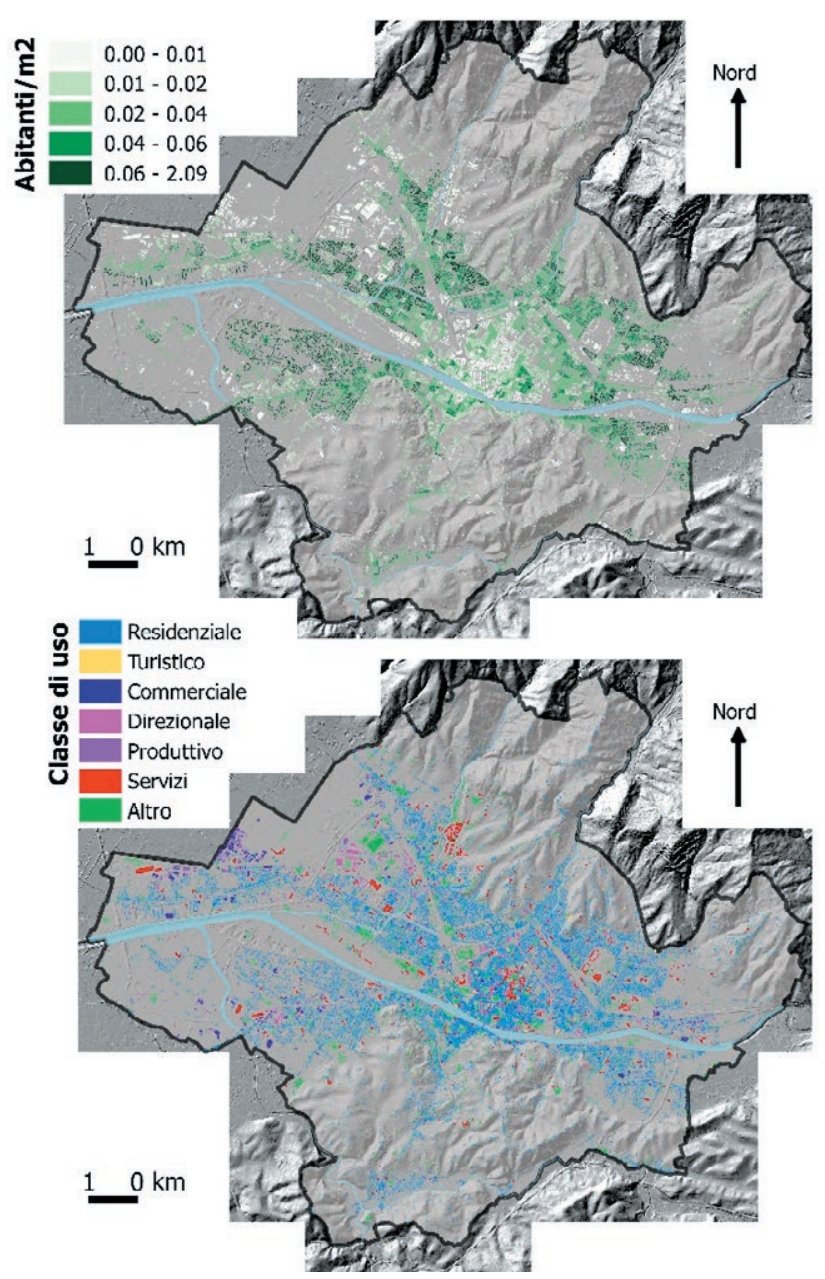

Figura 1. Mappe della distribuzione degli abitanti (a) e delle classi di uso dell'edificato dell'area fiorentina (b). Fonte: Elaborazione Pauline Deguy.

c) dal 1924 al 1955: muratura a sacco con fondazione diretta (27\%);

d) dal 1956 al 1978: telaio in CA e fondazioni a trave o a plinti (25\%);

e) dopo il 1978: struttura in CA, conforme alle varie leggi antisismiche dopo il 1982 (3\%).

Relativamente ai 31.070 edifici residenziali che oggi sono presenti nella città di Firenze, si possono fare le seguenti osservazioni:

1) circa il $58 \%$ era già presente nel 1895 , l'anno dell'ultimo "grande terremoto": si tratta degli edifici che si trovano nelle aree che attualmente fanno parte del centro storico e nelle zone di espansione residenziale ottocentesche. Questi edifici possono aver subito nel tempo interventi più o meno invasivi di ristrutturazione (che potrebbero averne alterato la risposta comportamentale in caso di nuovo sisma);

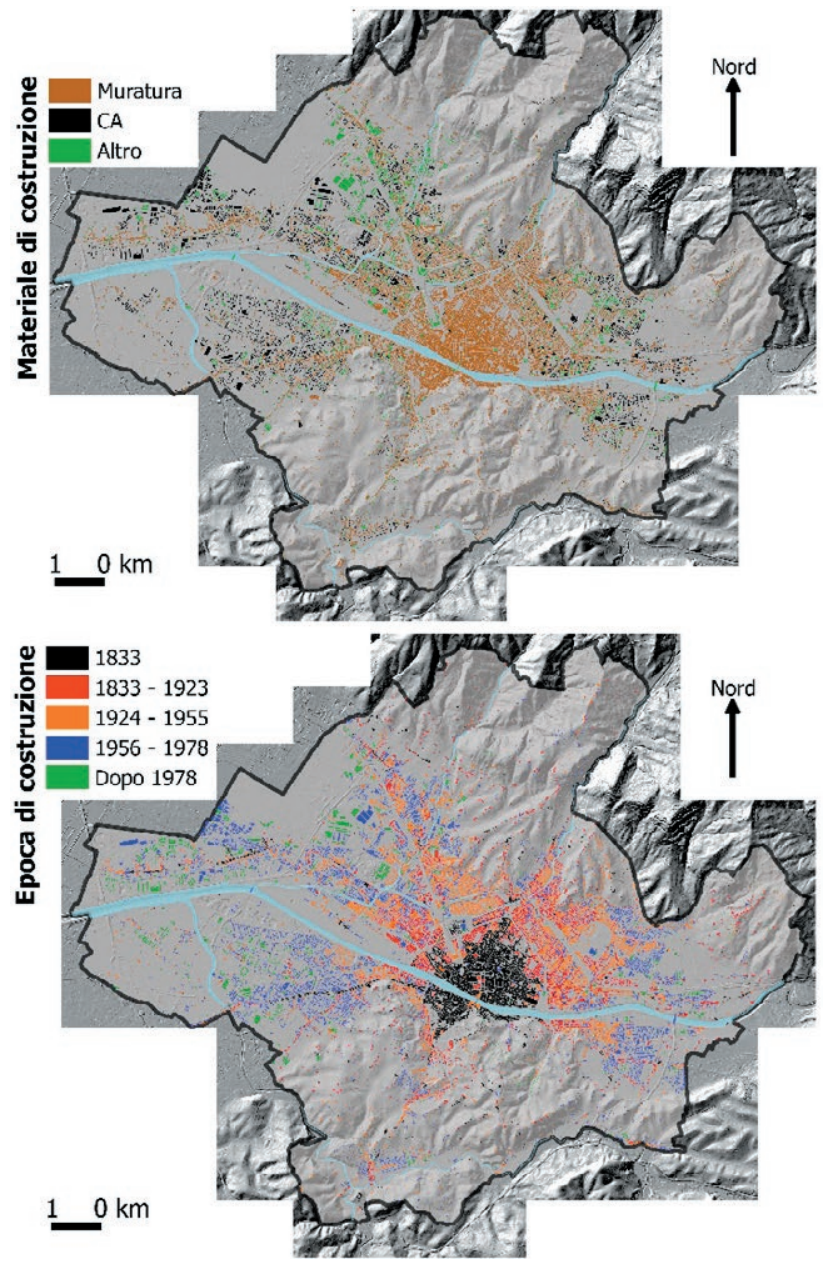

Figura 2. Mappe di distribuzione del materiale (a) e dell'epoca di costruzione dell'edificato fiorentino (b). Fonte: Elaborazione Pauline Deguy, dati Ufficio Urbanistica del Comune di Firenze.

2) circa il $40 \%$ è stato costruito tra il 1895 e il 1981, è stato quindi edificato in assenza di vere e proprie normative antisismiche, e non ha mai subito il "collaudo" di un terremoto; pertanto una quota consistente di questi edifici potrebbe rivelare una vulnerabilità maggiore rispetto a quelli ottocenteschi.

\section{Valutazione a larga scala della vulnerabilità sismica}

La vulnerabilità sismica dell'edificato di Firenze può essere rappresentata in maniera speditiva attraverso la probabilità della presenza o meno del fenomeno di risonanza, cioè la vicinanza dei modi fondamentali di oscillazione dell'edificio con quelli di amplificazione sismica al suolo. Nel caso in cui il periodo proprio di oscillazione della struttura e quello del suolo siano molto simili, 
vi è alta probabilità di osservare un fenomeno di risonanza che amplifica il movimento di oscillazione dell'edificio aumentando il rischio di danneggiamento ${ }^{4}$.

Il fattore fondamentale che caratterizza il periodo di oscillazione di un edificio è la sua altezza. Il fenomeno della risonanza è stato infatti scoperto dall'analisi dei danni condotta dopo il devastante terremoto del Messico del 19 settembre 1985: gli edifici che riportarono danni maggiori e collassi avevano un'altezza compresa fra 6 e 15 piani, mentre gli edifici con altezza inferiore o superiore subirono danni minori.

Le misure sismiche realizzate in-situ su un campione di edifici di Firenze hanno permesso di definire una legge del periodo proprio di oscillazione delle strutture in muratura in funzione della loro altezza (Ripepe et al. 2015; Ripepe et al. 2016). Per quanto riguarda le strutture in cemento armato, sono state usate le prescrizioni dalla normativa per le strutture (NTC 2008). In base alla mappa del periodo del suolo e a quella dei periodi delle strutture (Fig. 3), sono state realizzate delle mappe dell'indice di risonanza $(I R)$ per gli edifici in muratura e per quelli in cemento armato applicando la seguente formula:

$I R=\frac{1}{1+\sqrt{(1-T S / T e)^{2}}}$

dove $T s$ è il periodo relativo alla massima amplificazione sismica prodotta dal sottosuolo e Te è il primo modo di oscillazione dell'edificio.

I valori ottenuti sono stati quindi suddivisi in 5 classi in modo da poter rappresentare il livello di vulnerabilità (Tab. 2). Sotto un valore di $I R=0,7$, il livello di vulnerabilità è considerato da molto basso a basso, per i valori di $I R$ compresi tra 0,7 e 0,8 la vulnerabilità è media, mentre per $I R>0,8$ il livello di vulnerabilità è alto, fino ad arrivare a molto alto per valori oltre 0,9 . Per rappresentare il livello di vulnerabilità è stata calcolata la media dei valori ottenuti per ogni singolo edificio all'interno di una griglia con celle di 100x100 metri di lato, facendo una distinzione tra muratura e cemento armato. Questo valore, considerato come rappresentativo della cella, è stato quindi utilizzato per realizzare le mappe di vulnerabilità del tessuto urbano (Fig. 4).

\subsection{Esposizione della popolazione residente}

L'esposizione al rischio della popolazione residente è stata valutata a partire dall'analisi della sua distribuzione territoriale e dalle informazioni demografiche ISTAT

\footnotetext{
${ }^{4}$ https://youtu.be/LV_UuzEznHs
}

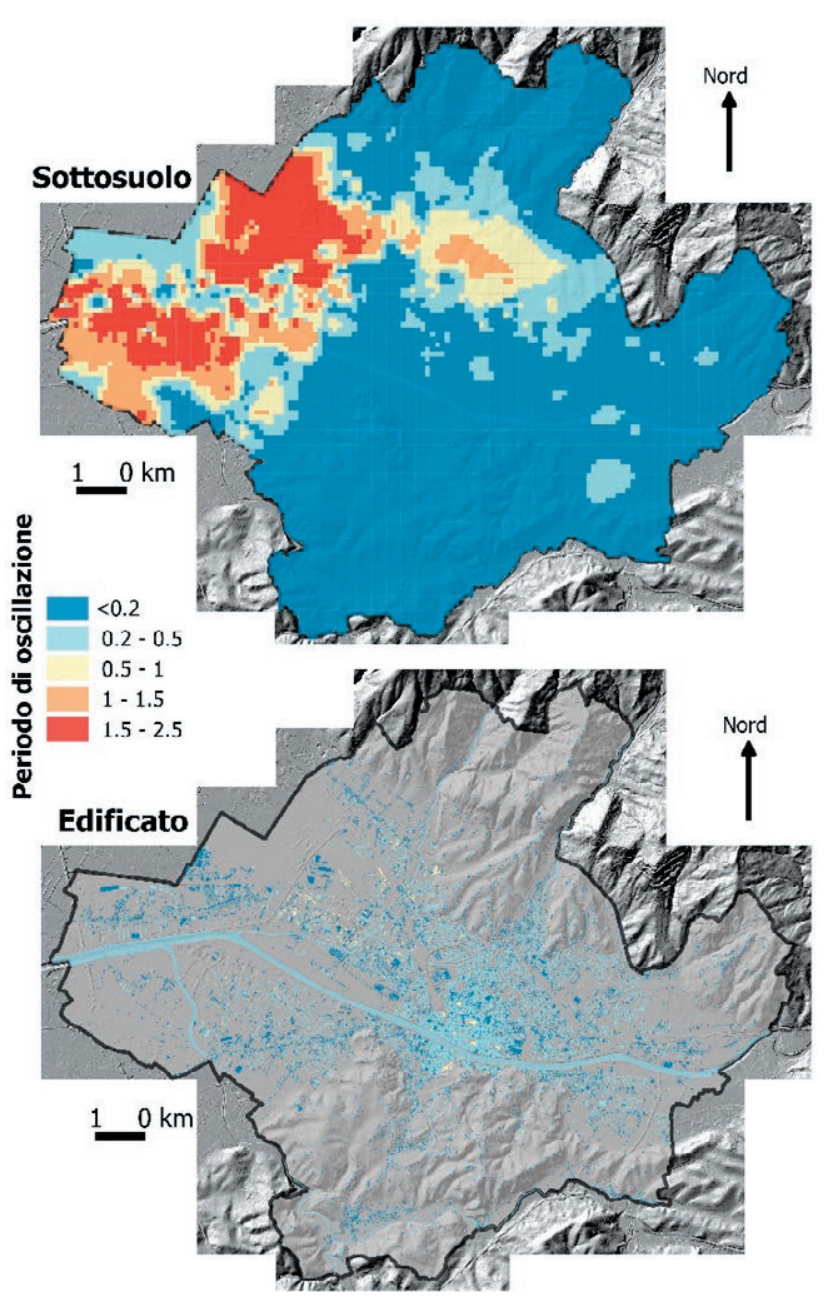

Figura 3. Mappe dei periodi di oscillazione del sottosuolo (Ts) (a) e delledificato (Te) fiorentino (b). Fonte: Elaborazione Pauline Deguy.

2011: sono state così definite le zone più esposte in termini di vite umane. L'esposizione è stata inoltre valutata in base a classi di età, come definite all'interno dei dati ISTAT (Corradi et al. 2014): da 0 a 14 anni, da 15 a 64 anni, sopra i 65 anni. La prima e la terza classe sono quelle ritenute più vulnerabili in caso di calamità trattandosi di persone che richiedono assistenza (popolazione dipendente). Le mappe di esposizione della popolazione sono state realizzate utilizzando queste due categorie (Fig. 5).

Oltre alla distribuzione della popolazione nel territorio, le mappe in figura 5 rivelano che il numero di individui con età maggiore di 65 anni è molto più elevato rispetto al numero di individui con età tra 0 e 14 anni: 92.378 individui (26\% del totale) contro 41.512 individui (11,6\% del totale), secondo i dati ISTAT.

La scelta di queste classi come quelle maggiormente esposte si basa sul potenziale comportamento e sulla 
Tabella 2. Classi di vulnerabilità.

\begin{tabular}{lc}
\hline Indice di risonanza & Livello di vulnerabilità \\
\hline$\square<0,6$ & Molto Basso \\
$\square>_{0,6}$ a 0,7 & Basso \\
$\square, 7$ a 0,8 & Medio \\
$\square, 8$ a 0,9 & Alto \\
$\square>0,9$ & Molto Alto \\
\hline
\end{tabular}

probabile reazione ad un evento calamitoso degli individui in funzione della loro età. Nella pianificazione del rischio è importante quindi considerare le zone di concentrazione della popolazione più vulnerabile per una migliore e più mirata organizzazione degli interventi di evacuazione o di assistenza medica e psicologica (Corradi et al. 2014).

Il rischio sismico è stato quindi analizzato per celle confrontando il livello di vulnerabilità (riferito all'indice di risonanza) e la classe di età degli abitanti presenti. Le mappe in figura 6 rappresentano rispettivamente l'indice di risonanza per gli edifici in muratura nelle zone con maggior concentrazione (almeno 50 individui per area) di giovani e di persone anziane. Per la classe di età inferiore a 14 anni, 146 celle $\left(1.460 .000 \mathrm{~m}^{2}\right)$, cioè il 9,6\% delle 1.544 celle presenti nella mappa, hanno un valore dell'indice di risonanza superiore o uguale a 0,8 , che corrisponde ad un livello di vulnerabilità da alto a molto alto. Per la classe di età superiore a 64 anni, sono 437 celle $\left(4.370 .000 \mathrm{~m}^{2}\right)$, cioè il $12,6 \%$ delle 3.437 celle presenti nella mappa, ad avere un livello di vulnerabilità da alto a molto alto.

\subsection{Esposizione della popolazione turistica}

Tra tutte le varie attrazioni turistiche del centro storico fiorentino, è stato possibile ottenere dati sugli ingressi mensili solamente per i musei comunali: Palazzo Vecchio, Torre di Arnolfo, Torre di San Niccolò, Cappella Brancacci, Basilica di Santa Maria Novella, Museo del Novecento, Fondazione Salvatore Romano, Museo Stefano Bardini e Forte Belvedere.

Per questi musei, tra il 2013 e il 2015, il numero totale di visitatori è passato da 1.223 .941 a 1.615 .684 , è aumentato cioè del $32 \%$. Dal grafico in figura 7 possiamo osservare che l'incremento maggiore si ha tra maggio e settembre, in corrispondenza cioè della cosiddetta alta stagione turistica; negli altri mesi dell'anno il numero di visitatori è rimasto mediamente stabile nei vari anni. Analizzando il numero di visite per singolo

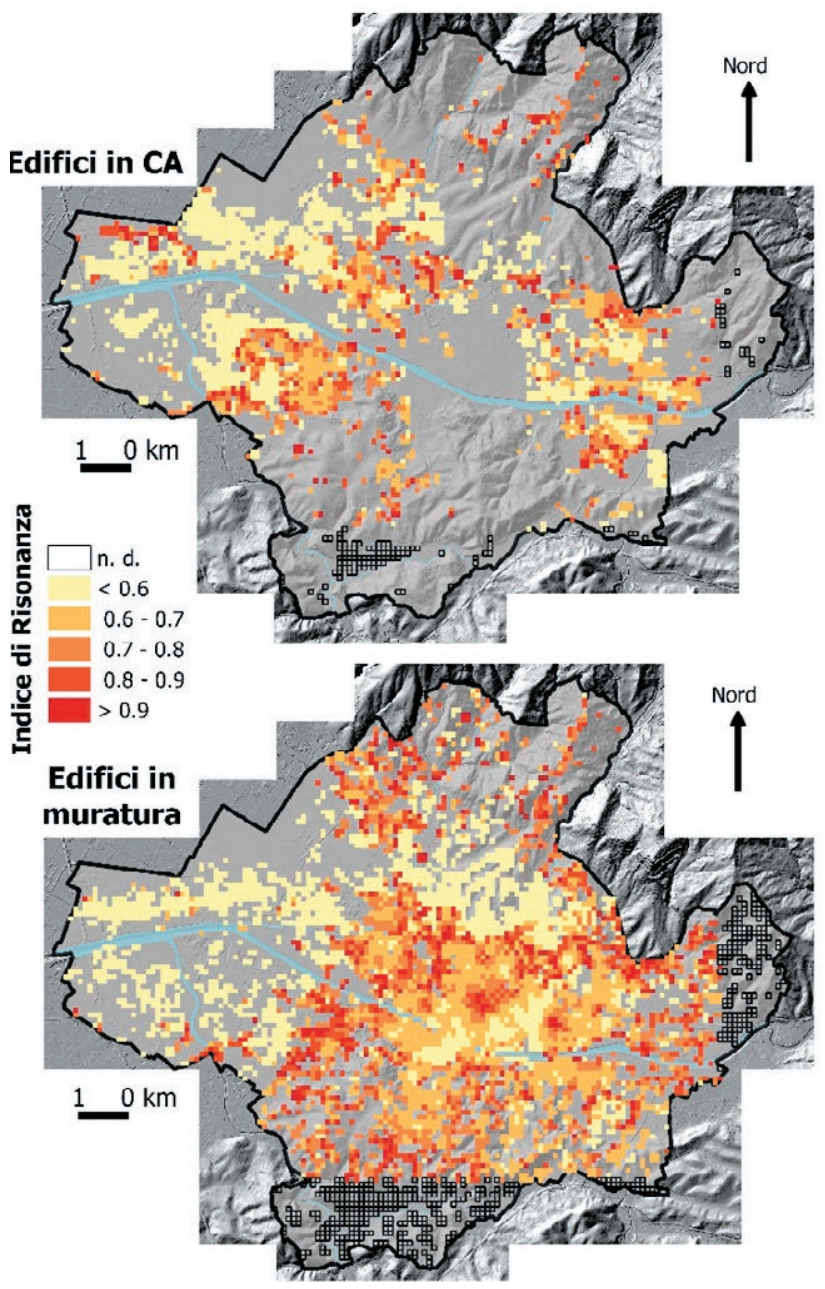

Figura 4. Mappe della distribuzione dell'indice di risonanza per gli edifici in cemento armato (a) e in muratura (b). Fonte: Elaborazione Pauline Deguy.

museo, si può sicuramente concludere che le strutture più sensibili sono Palazzo Vecchio e la chiesa di Santa Maria Novella.

Sempre al fine di conoscere gli elementi di alta vulnerabilità umana, dalla classificazione dei musei in funzione dell'affluenza annuale del pubblico, si osserva che le strutture più sensibili, a causa della notevole concentrazione di turisti (oltre 1 milione di visitatori all'anno), sono la cattedrale di Santa Maria del Fiore, la Galleria degli Uffizi, la Galleria dell'Accademia e Palazzo Pitti (Fig. 8).

Va considerato che questi dati non tengono conto del turismo giornaliero o di passaggio che riguarda $\mathrm{i}$ turisti presenti a Firenze per un giorno solo. Per stimare questa tipologia di turismo è stato rappresentato il numero di visitatori presenti all'interno dei musei e dei 

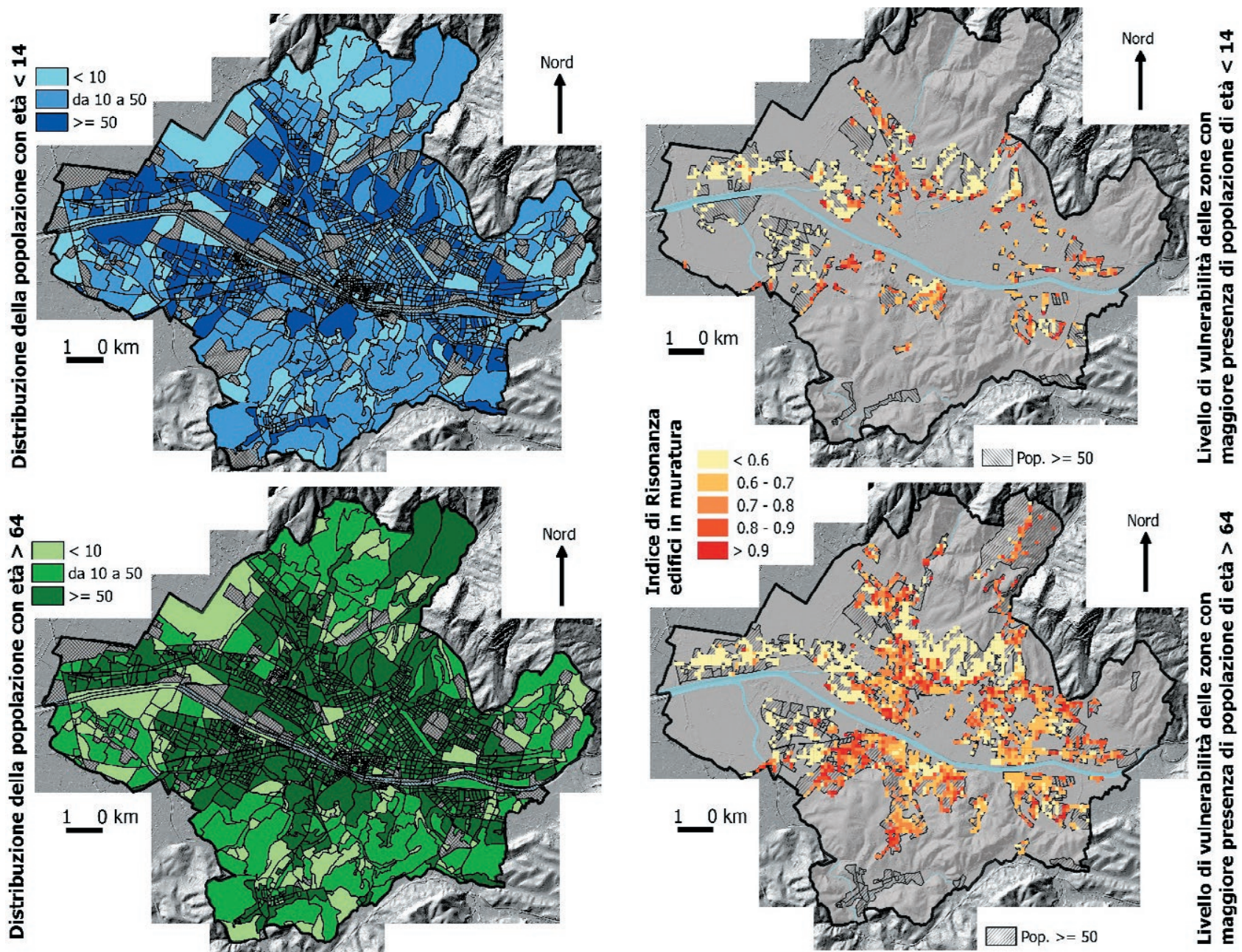

Figura 5. Rappresentazione, in scala cromatica, del numero di persone presente per area, delle due classi di età più vulnerabili (di età $<14$ (a) e di età > 64 (b)). Fonte: Elaborazione Pauline Deguy, dati Istat, 2011.

Evoluzione degli ingressi totali nei musei civici fiorentini

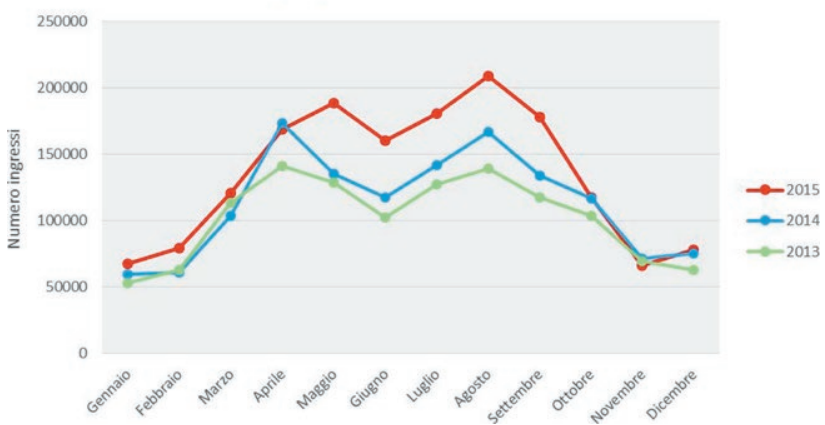

Figura 7. Evoluzione del numero totale di visitatori per mese nei musei comunali dal 2013 fino al 2015. Fonte: Elaborazione Pauline Deguy, dati Istat, 2011.
Figura 6. Mappe rappresentative del livello di vulnerabilità per le aree di maggior concentrazione di classi di giovani (a) e di persone anziane (b). Fonte: Elaborazione Pauline Deguy.

Evoluzione degli ingressi per ogni museo civico fiorentino

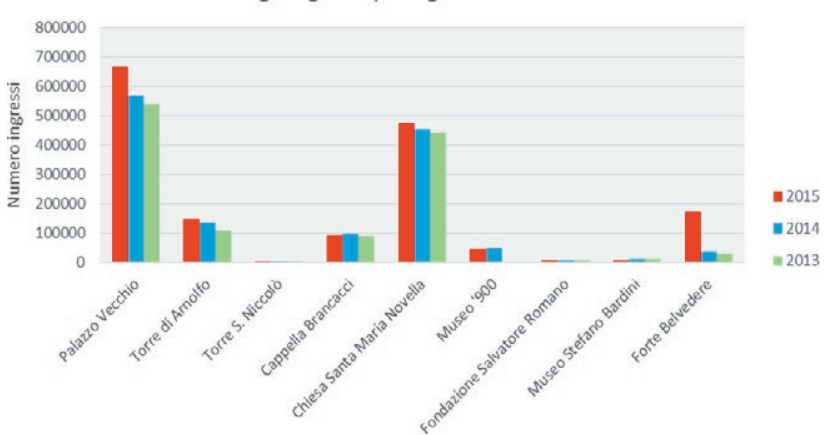

Figura 8. Evoluzione del numero di visitatori per museo civico dal 2013 al 2015. Fonte: Elaborazione Pauline Deguy, dati Istat, 2011. 


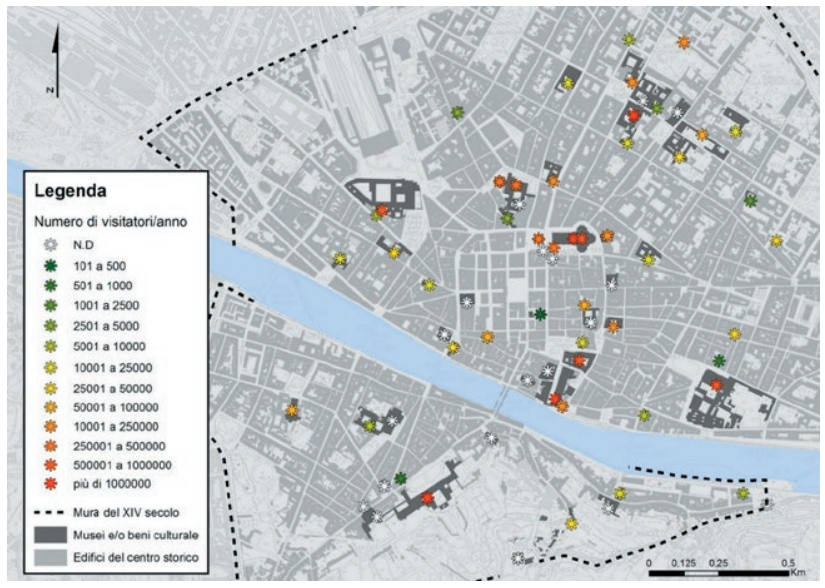

Figura 9. Affluenza del pubblico nei principali musei del centro storico fiorentino. Fonte: Elaborazione Pauline Deguy, dati Istat, 2011.

beni culturali (Fig. 9). Le informazioni trovate sono relative ai dati Istat del 2011. I musei del centro sono stati classificati a seconda dell'affluenza del pubblico durante l'anno. Per quattro di questi, il numero di visite annue va oltre il milione: la Basilica di Santa Maria del Fiore, gli Uffizi, la Galleria dell'Accademia e Palazzo Pitti.

Come già introdotto nel paragrafo 2.1, per valutare il livello di esposizione al rischio della popolazione turistica, maggiormente concentrata nel centro storico, dove si trova la quasi totalità dei beni culturali e delle strutture ricettive, è fondamentale conoscere la destinazione d'uso degli edifici: i beni culturali sono le strutture più sensibile all'esposizione umana durante le ore diurne, mentre le strutture ricettive sono chiaramente più esposte durante la notte.

\section{Conclusioni e prospettive}

La presente ricerca ha condotto alla realizzazione di una metodologia per la valutazione del rischio sismico e a l'implementazione delle informazioni utili a tale scopo nella città di Firenze. La tecnologia GIS è stata usata al fine di individuare le relazioni spaziali di un sistema complesso e di analizzarne tutte le componenti a rischio.

Partendo dalla formula del rischio, è stata analizzata la pericolosità attraverso l'uso di dati geofisici e geologici prendendo in considerazione il valore del periodo delle onde sismiche e il profilo stratigrafico del sottosuolo. L'esposizione, o valore esposto, è stato trattato con l'uso di strumenti GIS per arrivare a creare un database capace di contenere l'insieme delle informazioni raccolte e di cartografarle. Le mappe così realizzate permettono di identificare in maniera semplice e speditiva le zone del- la città più esposte al rischio sismico: i quartieri, o gli isolati, con una concentrazione maggiore di abitanti, gli edifici costruiti con materiale meno resistente e il loro uso predominante. La vulnerabilità degli edifici è stata valutata dal punto di vista dell'interazione della struttura con il sottosuolo, mentre quella umana è stata trattata utilizzando dati ISTAT allo scopo di identificare le zone con una presenza maggiore di individui considerati più vulnerabili: i giovani sotto i 14 anni e le persone oltre i 65 anni.

La metodologia usata per definire la vulnerabilità delle strutture ha condotto alla creazione di un indice di risonanza al quale è stato associato un livello di vulnerabilità diviso in 5 classi, da molto basso a molto alto. Poiché il rischio sismico è il risultato dell'equazione Pericolosità x Vulnerabilità x Valore esposto, al fine di giungere alla sua definizione, nell'ultima fase del progetto sono state confrontate le mappe di risonanza con quelle relative alla distribuzione territoriale della popolazione per fasce di età. La metodologia da noi proposta permette, in maniera speditiva, di definire e di valutare le zone con una percentuale di rischio più elevata fornendo indicazioni preziose sulla fragilità del territorio e sulle zone dove intervenire per ridurre l'esposizione dei cittadini.

Una volta standardizzati i parametri di applicazione utilizzati nel presente lavoro, questa metodologia potrebbe essere applicata ad altri ambiti territoriali come strumento di supporto alla decisione nella realizzazione dei Piani Comunali di Emergenza. Utilizzando il sistema e le metodologie sviluppate in questo studio, opportunamente adattate, sarebbe possibile strutturare piani di prevenzione e/o di salvaguardia da altri rischi ambientali, quali quelli derivanti da esondazione o da allagamento. Nel caso della creazione di un sistema informativo territoriale per un altro territorio, la tipologia delle informazioni da raccogliere, la metodologia di rilievo e di analisi dei dati sarebbero simili, così come le competenze necessarie per la sua realizzazione.

\section{Riferimenti bibliografici}

Bertin, M. (2015). La riduzione del rischio sismico in ambiente urbano. In Carnelli, F., Ventura, S. (a cura di). Oltre il rischio sismico. Valutare, comunicare e decidere oggi. Roma, Carocci, 61-86.

Calvi, G.M., Pinho, R., Magenes, G., Bommer, J.J., Restrepo-Vélez, L.F., Crowley, H. (2006). Development of seismic vulnerability assessment methodologies over the past 30 years. ISET Journal of Earthquake Technology, 43 (3), 75-104. 
Coli, M., Ripepe, M., Rubellini, P. (2008). Firenze, sismicità dellarea fiorentina. Firenze, SELCA.

Coli, M., Rubellini, P. (2007). Firenze, note di geologia fiorentina. Firenze, Firenze, SELCA.

Coli, M., Pini, G., Rubellini, P. (2007). Firenze: carta litotecnica del territorio comunale. Firenze, SELCA.

Coli, M., Dinoi, G., Lacanna, G., Marchetti, E., Pini, G., Ripepe, M., Rubellini, P. (2008). Firenze: carta sismica del territorio comunale. Firenze, SELCA.

Corradi, J., De Fausti, F., Salvucci, G., Vitale, V. (2014). Popolazione e vulnerabilità sismica. Giornate della ricerca in ISTAT (10-11 novembre), sessione IV: Metodologie di sintesi e analisi del territorio. Roma, ISTAT.

https://www.istat.it/it/files/2014/10/Paper_Sessione-IV_ Corradi_De-Fausti_Salvucci_Vitale.pdf

Cremonini, I. (a cura di). (2004). Analisi, valutazione e riduzione dell'esposizione e della vulnerabilità sismica dei sistemi urbani nei piani urbanistici attuativi. Regione Emilia-Romagna, Direzione generale Programmazione territoriale e sistemi di mobilità, Servizio Riqualificazione urbana, Bologna.

Cutter, S., Mitchell, J., Scott, M. (2003). Revealing the Vulnerability of people and places: A case Study of Georgetown Country, South Carolina. Annals of the Association of American Geographers, 90 (4), 713-737.

Deguy, P. (2017). Valutazione e rappresentazione della ricaduta sociale e sul patrimonio artistico della vulnerabilità sismica valutata a grande scala e relativamente agli edifici storici della città di Firenze, Tesi di dottorato, Università degli Studi di Roma “Tor Vergata”, Italia.

Comune di Firenze, Direzione Sistemi Informativi Servizio Statistica e Toponomastica (2013). Bollettino mensile di Statistica. Firenze.

NTC (2008). Nuove Tecniche per le costruzioni. D.M 14 gennaio 2008, Gazzetta Ufficiale $n^{\circ} 29$ del 4 febbraio 2008.

NTC (2018). Nuove Tecniche per le costruzioni. D.M. 17 gennaio 2018, Gazzetta Ufficiale $\mathrm{n}^{\circ} 8$ del 20 febbraio 2018.

Pesaresi, C. (2010). Il contributo dei GIS nell'analisi del rischio sismico e vulcanico. In Bozzato, S. (a cura di). GIS tra natura e tecnologia. Strumento per la didattica e la diffusione della cultura scientifica. Roma, Carocci editore, 173-186.

Regione Umbria (2013). Rischio sismico urbano. Indicazioni di metodo e sperimentazioni per l'analisi della Condizione limite per l'emergenza e la Struttura urbana minima.
Rapporto finale di ricerca (DGR Umbria $n^{\circ} 793$ del 18 luglio 2011, determinazione dirigenziale $n^{\circ} 3928$ del 23 maggio 2012).

Ripepe, M., Lacanna, G., Deguy, P., De Stefano, M., Mariani, V., Tanganelli, M., (2015). Large-scales seismic vulnerability assessment method for urban centres. An application to the city of Florence. Key Engineering Materials, 628, 49-54.

Ripepe, M., Lacanna, G., Deguy, P., Coli, M., Paoletti, B., Barducci, S., Viti, S., De Stefano, M., Tanganelli, M. (2016). Seismic Hazard of Urban Areas: A case-study. Atti del convegno ECCOMAS Congress 2016: VII European Congress on Computational Methods in Applied Sciences and Engineering, Hersonissos, Greece.

Silei, G. (a cura di) (2011). Ambiente, rischio sismico e prevenzione nella Storia d'Italia. Piero Lacaita Editore.

Varnes, D.J., IAEG Commission on Landslides (1984). Landslide Hazard Zonation - a review of principles and practice. Paris, UNESCO. 